\title{
Restaurante típico y familiar de una región turística: Un estudio de caso sobre el impacto de la marca en la fidelidad del cliente
}

\author{
Hamilton Pozo \\ FACCAMP, Faculdade Campo Limpo Paulista (Brasil) \\ Roselaine Aparecida de Faria Teodoro ${ }^{\text {ii }}$ \\ UNIESP (Brasil) \\ Takeshy Tachizawaiii \\ FACCAMP, Faculdade Campo Limpo Paulista (Brasil)
}

\begin{abstract}
Resumen: El objetivo de esta investigación es analizar el efecto de la personalidad de la marca de un restaurante en las emociones del cliente (positivas y negativas), la satisfacción del cliente y la fidelidad a la marca, utilizando modelos de ecuaciones estructurales. La investigación también analiza la aplicabilidad del modelo fivefactor en la escala de personalidad de la marca para restaurante. Los datos empíricos fueron colectados con la aplicación de cuestionarios en seis restaurantes típicos de Campos de Jordão-SP. Esta investigación confirma cinco dimensiones de la personalidad típica de un restaurante con enfoque turístico. También, los resultados sugieren que las emociones de los clientes impactan directamente en la satisfacción y fidelidad a la marca. De esta forma, los emprendedores ganarán mucho ante un acompañamiento constante de las percepciones de sus clientes en relación a la marca y a la personalidad del restaurante.
\end{abstract}

Palabras clave: Personalidad de la marca; Restaurante; Emoción; Satisfacción del cliente; Fidelidad.

Title: Typical family restaurant and tourist area: A case study on the impact of the brand in customer loyalty

\begin{abstract}
The objective of this research is to analyze the effect of brand personality of a restaurant in the client's emotions (positive and negative), customer satisfaction and brand loyalty, using structural equation models. The research also examines the applicability of the model in the fivefactor in brand personality scale for a restaurant. Empirical data were collected with the use of questionnaires in six typical restaurants of Campos de Jordão-SP. This research confirms five personality dimensions typical of a tourist-focused restaurant. Also, the results suggest that emotions directly impact customer satisfaction and brand loyalty. In this way, entrepreneurs will gain much with a constant accompaniment of the perceptions of customers regarding the brand and the personality of the restaurant.
\end{abstract}

Keywords: Brand personality; Restaurant; Emotion; Customer satisfaction; Loyalty.

i $\mathrm{PhD}$ en Administración e profesor e investigador de la Maestría en Administración de Empresas en el ámbito de la Micro y Pequeña Empresa em La Faculdade Campo Limpo Paulista-Faccamp (Brasil). E-mail: h.pozo@ faccamp.br

ii estudiante de posgrado em Administración e profesor de la responsabilidad social em UNiesp (Brasil). E-mail: faria@federal.zzn.com

iii Doutor en Administración e profesor e investigador de la Maestría en Administración de Empresas en el ámbito de la Micro y Pequeña Empresa em La Faculdade Campo Limpo Paulista-Faccamp (Brasil). E-mail: usptakes@faccamp.br 


\section{Introducción}

Los clientes muchas veces toman decisiones sobre restaurantes con base en diversos atributos, como localización, la cualidad de la comida, servicio, precio, experiencias anteriores y marca (Jiang, Dev; Rao, 2002). Recientemente, las investigaciones empezaron a analizar el efecto de la personalidad de la marca percibida por los clientes en la utilización de los servicios por medio del comportamiento de estos (Fournier, 1994; Yik, 2001). La personalidad de la marca es definida como un conjunto de atributos semejantes a la personalidad de los seres humanos asociados a un tipo particular (Aaker, 1997). Aaker (1996) sugiere que la personalidad de la marca puede proveer un link entre los beneficios emocionales y auto-expresión de un producto y el servicio prestado que pasa a ser considerado como base para mejorar el relacionamiento con los clientes, creando una diferenciación de marca. Matthies (1997) sugiere también que la personalidad de la marca puede aumentar el valor agregado del servicio y la ampliación de esa marca. Y a su vez, Randall (1997) apresenta cuatro principales funciones de la personalidad de la marca:

a) ofrecer identidad al cliente;

b) hacer una base de datos con todas las informaciones ofrecidas por el cliente sobre el servicio;

c) garantizar la tranquilidad esperada y los beneficios;

d) acrescentar informaciones sobre el valor del servicio prestado.

Recientes investigaciones están centradas sobre esos datos relacionados a la personalidad de la marca y a la emoción en el proceso de consumo (Biel, 1993; Donovan; Rossiter, 1982; Yik, 2001). Biel (1993) afirma que la personalidad de la marca evoca emociones em los clientes, lo que es consistente con la conclusión de Yik (2001), de que las acciones más duraderas afectan la personalidad y las influencian, y estas, a su vez, conducen al comportamiento de satisfacción del cliente. Aaker (1996) afirma que la personalidad de la marca provee un beneficio emocional a partir del momento em que los clientes piensan en utilizar el servicio (la marca). El estado emocional positivo de los clientes irá influenciar positivamente el número de itenes comprados, el tiempo utilizado en la tienda, y el valor monetario gastado en la tienda (Donovan; Rossiter, 1982). Considerando, que la emoción es un antescedente a la satisfacción del cliente (Babin; Darden, 1996; Desai; Mahajan, 1998).

El comportamiento del consumidor es retratado como un estímulo a un sistema de respuesta del organismo. Estos estímulos son "externos a la persona" y compuesto por dos variables el mix de marketing y de otros insumos ambientales. Por lo tanto, la personali- dad de la marca puede ser usada como estímulo para que los emprendedores puedan incentivar a los clientes en sus aptitudes y acciones y de esa forma, aumentar los niveles de la personalidad de la marca y de la lealtad (Fournier, 1994). Así, es muy importante que los emprendedores de restaurante típicos sepan más sobre el comportamiento de sus clientes y como estos pueden ser influenciados por la personalidad de la marca y por sus necesidades emocionales.

Mismo que la personalidad de la marca y la emoción generada por ella sean reconocidas en la literatura existente como factores importantes en el marketing de relacionamiento, poca investigación empírica fue realizada sobre los efectos de la personalidad de la marca en los clientes en el comportamiento de la post compra. Este estudio investiga la personalidad de la marca de un restaurante y su papel en la creación de valor para la satisfacción del cliente y su consistente fidelidad a través del papel mediador de las emociones del cliente. Este estudio también abarca como las variables de personalidad de la marca, tales como sinceridad, entusiasmo, competencia, cualidad, sofisticación, servicio pueden afectar el estado emocional del cliente. Es importante resaltar que los resultados de este estudio tienen implicaciones teóricas y prácticas. Las implicaciones teóricas incluyen un mejor entendimiento de los factores de la personalidad de la marca sobre el estado emocional de los clientes dentro del sector.

Tal actitud ofrece informaciones y los más ricos insights sobre la estructura y los procesos de marketing de la teoría de la marca. Con respecto a la aplicación práctica, los emprendedores pueden desarrollar estrategias eficaces de posicionamiento, investigando la relación entre la personalidad de la marca y la fidelidad a la marca, medida por factores emocionales, y como ésta se refleja en la satisfacción del cliente. Es fundamental para que los emprendedores de restaurantes típicos puedan evaluar los factores que contribuyen para la creación de experiencias agradables y/o desagradables que puedan afectar el comportamiento de los actuales clientes y los en potencial.

\section{La personalidad de la marca de un restaurante típico}

Trazos de personalidad son determinados a partir de la observación y del comportamiento, bien como características físicas (Park,1986). Así, como las personas desarrollan sus proprias personalidades, reflejando así un proceso relativamente sencillo de desarrollo de la personalidad (McCrae et al., 2000). De la misma forma se define la personalidad de la marca como la inmediata 
manera emocional que se obtiene como respuesta de las personas a una marca (Larson, 2002). Segundo Burke (1994), una estrategia eficaz usa la personalidad de la marca, correctamente y consistentemente comunicada $\mathrm{y}$, por lo tanto, otorga una impresionante variedad de beneficios, incluyendo los siguientes:

1. la construcción de una marca propia generando un posicionamiento en la aprovación general;

2. la creación de un reconocido ambiente para una comunicación eficaz con los clientes;

3. el desarrollo de una fuerte imagen de la marca para los productos y servicios prestados;

4. la diferenciación de la marca en el mercado;

5. permitir a la empresa un valor agregado altamente percibido para sus productos y servicios.

Aaker (1997) desarrolló una escala para la personalidad de la marca (EPM) - Brand Personality Scale [BPS] - para explorar el significado de 37 marcas comerciales, examinando como esos atributos de la personalidad están estructurados en la mente de las personas en los Estados Unidos. El desarrollo del proceso aseguró la validad, confiabilidad y generalización de la escala en el ámbito de las marcas. Un total de 42 trazos de personalidad EPM fueron identificados en cinco dimensiones: sinceridad, entusiasmo, competencia, robustez y sofisticación. Estas cinco dimensiones han descrito con exito las personalidades de muchas marcas fuertes en los Estados Unidos (Aaker, 1996). Por ejemplo, una marca puede tener una personalidad compleja, que varía en dimensiones, es el caso de McDonald's que fue evaluada con elevado atributo en sinceridad y competencia. En un aplicativo EPM de Aaker en segmentos diferentes de restaurante, tales como: fast food, de cenas ocasionales y de almuerzo ejecutivo fueron distinguidos con base en las cinco dimensiones de la EPM, pero, en relación a la distinción entre los restaurantes de lujo la escala EPM no tuve exito (Siguaw, Mattila; Austin, 1999).

\section{Las emociones}

Las emociones de una persona pueden ser positivas y negativas. Las personas en estados emocionales positivos tienden a disminuir la complejidad de su tomada de decisión y tienen menos tiempo de decisión que las personas en estados emocionales negativos. Investigaciones han mostrado que las emociones contribuyen significativamente para la selección de prestadores de servicios, la evaluación de la cualidad del servicio, la determinación en repetir los comportamientos de compra y el desarrollo de fidelidad a la marca (Barsky; Nash, 2002; Mattila; Enz, 2002; Becker; Murrmann; Murrmann; Cheung, 1999). Hay que concentrarse en aspec- tos emocionales de la experiencia de consumo para una mejor comprensión de la evaluación en el proceso de atendimiento al cliente (Mattila; Patterson,2004). Dubé y Menon (2000, p.288) sugieren que las emociones son respuestas efectivas al consumo y a la propia percepción de los diversos atributos que componen la evidencia de un producto o servicio. Esas emociones son normalmente intencionales porque ellas tienen un objeto o referencia y que son diferentes del concepto de humor, que es un estado generalizado inducido por una variedad de factores y es normalmente cansativo y no intencional (Bagozzi, Gopinath; Nyer, 1999). En otras palabras, las emociones de consumo muchas veces exigen más intensidad, motivación, fuerza, uma situación específica que la urgencia psicológica del humor.

El componente emocional en la satisfacción es por lo tanto, independiente del sentido global de los afectivos regalos a los clientes en el período del tiempo de servicio (Ruyter; Bloemer,1999). En los estudios, geralmente, se identifican dos maneras diferentes de comprender las características subjacentes a la emoción del consumo, es decir, en la categoría y en la dimensión estructural (Oh, 2005). En el análisis de la categoría están presentes varios factores independientes monopolar (Oh, 2005). Por ejemplo, Plutchik (1984) desarrolló una escala de la emoción, identificando las categorias principales, incluyendo la aceptación, la alegría, la espectativa, el miedo, la cólera, la tristeza, la sorpresa y la repugnancia. Varias investigaciones han apoyado la confiabilidad y la validad de medir la emoción del consumo mediante las categorías de la emoción, fundamental en sus estudios (Batra; Holbrook, 1990).

El estudio de la dimensión estructural presupone que los estados emocionales están relacionados uno con el otro de forma sistemática, en vez de independientes uno del outro (Oh, 2005). Al contrario del estudio de la categoría, la dimensión estructural asegura la existência de una estructura bipolar de medidas. Como ejemplo, las emociones de consumo fueron medidas a través de una serie de escalas semánticas, como un continuo bipolar de placer, excitación y dominación. Varias investigaciones han apoyado el estudio de la categoría, presentando evidencias empíricas (Holbrook; Westbrook, 1990; OH, 2005). Además, Machleit y Eroglu (2000) afirman que las emociones positivas y negativas tienen variaciones únicas e influencia sobre el comportamiento de forma independiente y que los clientes son afectados por dos factores unipolares independientes que son dimensiones correspondientes al sentido positivo o negativo de una emoción de consumo (Babin, Darden; Babin, 1998; Lee, Lee; Lee, 2005; Yoo, Park; Macinnis, 1998). 


\section{La satisfacción del cliente}

La satisfacción del cliente es fundamental para el concepto de marketing como la noción de satisfacer las necesidades y deseos de los consumidores (Spreng, Mackenzie \& Olshavsky, 1996; Hsu, 2009). La definición de satisfación del cliente varía a lo largo de la literatura de marketing. La satisfacción del cliente en la percepción cognitiva del comprador puede ser adecuada o inadecuadamente recompensada por los sacrificios que él puede percibir. La definición de un resultado ante los sacrificios recibidos por medio de la experiencia del consumo (YI, 1990) y la satisfacción de los clientes, mismo como el resumen del estado psicológico resultante de la emoción en torno de las expectativas está asociada con el sentimiento previo del consumidor sobre la experiencia del consumo (Oliver 1992). Los resultados de la satisfacción del cliente cuando quiere confirmar su expectativa antes de la compra de un servicio o no confirmar positivamente sus expectativas a respecto de los servicios adquiridos, resultando en un nível de servicio después de la compra, afectan su experiencia en relación al proveedor.

La manera por la cual la satisfacción del cliente es medida ha sido discutida en términos de escalas que son utilizadas y en la forma de las preguntas (Wilson, 2002). La satisfacción del cliente puede ser medida por una o por varias alternativas. Por consecuencia, es aceptable, cuando las escalas multi alternativas son utilizadas, debe ser considerado que la satisfacción del cliente es complea, porque las escalas de múltiples alternativas pueden capturar más connocimiento sobre la satisfacción del cliente, a partir de la perspectiva del propio consumidor en vez de un único iten. Varios ítenes también pueden ofrecer confiabilidad en la escala empírica estable cuando es comparado con un único iten (Gilbert; Veloutsou, 2006).

La satisfacción del cliente también puede ser medida por dos tipos distintos de transacciones: satisfacción específica y satisfacción global. La primera está relacionada a un encuentro específico con objetos específicos, mientras la segunda es una construcción acumulativa totalizando satisfacción con los productos y servicios (Faria; Tachizawa, 2008). Yuksel, Kilinc y Yuksel, (2006) explican que la recuperación del servicio bien ejecutado puede aumentar la satisfacción del cliente y la lealtad; puede tener una influencia directa sobre si los clientes siguen insatisfechos con o defecto de una organización.

Por eso, satisfacción general puede basearse en muchas transacciones o en apenas algunas, dependiendo de la cuantidad de veces que el consumidor tenga usado un objeto específico. En otras palabras, la satisfacción global es un agregado de todas las avaliaciones anteriores específicas es actualizada después cada acción específica. En general, la satisfacción global es una media variable que es relativamente más estable que las transaciones específicas de satisfacción (Parasuraman, Zeithaml; Berry, 1994).

En este estudio, la satisfacción del cliente es medida en términos de satisfacción global. Grande parte de investigaciones sobre la fidelidad a la marca se concentra en definir y medir factores de esa fidelidad y de su asociación en relación de largo plazo entre compradores y vendedores. La fidelidad a la marca definida con base en creencia, actitud y estructura de la intención de los consumidores de una determinada marca conduce al cliente a un tipo especial de compromiso con ella. Este compromiso direcciona al cliente a comprar la misma marca cada vez que el mismo producto es usado. De acuerdo con Davidow y Uttal (1989), los clientes que son fieles a la marca ofreren a los proveedores una triple recompensa: el marketing y los costos de ventas para mantenerlos son muy inferiores a los de obtención de nuevos clientes, esa reducción en el costo de marketing lleva a la reducción del costo de transacciones y comunicaciones y, finalmente, los clientes fieles compran más que un cliente moderadamente fiel o un nuevo cliente.

\section{Desarrollo de las hipótesis}

Las relaciones entre la personalidad de la marca y las emociones del cliente. La personalidad es relativamente estable, al menos en la cuestión de largo plazo. La emoción es momentánea, o por lo menos de corto plazo y es una cuestión de consciencia y sentimentos accesibles (Yik, 2001). Muchos psicólogos se han centrado en la relación entre personalidad y emoción, relatos han demonstrado que la personalidad está relacionada a la emoción (Izard, Libero, Putnam, Haynes, 1993; Watson, Clark, 1997). Estudios también sugieren que los trazos de personalidad pueden ser duraderos y antescedentes de las propias emociones (Mccrae; Costa, 1991; Yik, 2001). En relación con esa evidencia, Biel (1993) sugiere que la personalidad de la marca evoca emociones en el comportamiento de los consumidores en contextos de marketing. Esto significa que una comprensión de naturaleza emocional va a depender del grado en que es más dependiente del contexto o de la personalidad dependiente (Yik, 2001).

Las relaciones entre las emociones del cliente, su satisfacción y la fidelidad a la marca. El concepto de emoción comprende una clase de fenómenos mentales y que singularmente se caracteriza por un sentimiento 
experimentado conscientemente de un estado subjetivo y, de modo general acompaña afecto y humor. La emoción se realiza y puede surgir como una función de la evaluación del individuo sobre el significado, las causas, las consecuencias y/o las implicaciones personales de un estímulo particular (Westbrook, 1987).

Varias investigaciones han demonstrado la relación entre la emoción del cliente, su consumo y su comportamiento. Morris, Woo, Geason y Kim (2002) consideran que la respuesta emocional es un poderoso receptor de las intenciones y de la atitud en relación a la marca, que es consistente en relación a los comportamientos de compra de los clientes que son fuertemente influenciados por sus emociones y que las emociones influyen en la satisfacción y patrocinio en la repetitividad de la compra. Por ejemplo, Han y Back (2007) encuentran una relación significativa entre las experiencias emocionales de los huéspedes y su constante regreso al mismo local de hospedaje.

Específicamente en este caso, la cena del restaurante del hotel fue fuerte influenciador en esta emoción. El cliente se focó, principalmente, en recibir una óptima comida con un servicio simpático, de modo a influenciar su emoción en relación a la personalidad y por ser altamente agradable y generar mucha satisfacción, acabó reforzando las emociones de los clientes. Así, las emociones son importantes componentes de respuestas para el cliente, influenciando en su satisfacción y patrocinando la repetición de la acción (Allen, Machleit; Kleine, 1992; Walsh, 2000; Laverie, Kleine; Kleine, 1993).

Oliver (1997) afirma que la satisfacción puede ser determinada usando el duplo proceso de un modelo cognitivo y afectivo. Clientes observan y experimentan el desempeño de los emprendedores de servicios turísticos y los evaluan para sus futuras acciones. Por ejemplo, el anfitrión de un restaurante recibe un cliente de manera amistosa y atenciosa, llamándolo por su nombre y ofreciéndole una bebida, mientras el cliente aguarda en la mesa. El cliente pasa a sentirse más confortable y la espera se torna más agradable y placerosa, creando así, una atmósfera que enfluye directamente en la satisfacción afectiva del cliente. Esto significa que los clientes prueban una situação especial del servicio e implica, también, que viviendo un afecto positivo en un restaurante, el consumidor estará dispuesto a repetir la experiencia y, por lo tanto, se torna fiel al restaurante.

Yu e Dean (2001) sugirieron que hay una alta correlación entre la fidelidad de los clientes y los componentes emocionales y emociones positivas. Los estudios sugieren que la emoción enfluye en la retención de los clientes y en la vonluntad de comprar (Baker, Grewal; Levy, 1992).
La relación entre la satisfacción de los clientes y la fidelidad a la marca mostradas en las investigaciones, tradicionalmente, han afirmado la evidencia empírica de una relación positiva entre la satisfacción del cliente y la fidelidad a la marca. Bitner (1990) afirmó que la satisfacción del cliente tiene un efecto indirecto sobre la fidelidad de la marca. Rust y Zahorik (1993), también, encuentran una relación directa entre la satisfacción y fidelidad de los clientes a la marca en dos diferentes categorías de servicios, o sea, en los bancos y en una cadena hotelera americana. Albarracin y Wyer (2000), posicionan que el refuerzo del nivel de afecto positivo dado a los clientes aumenta las intenciones de recompra.

Con base en esa propriedad, Eagly y Chaiken (1993) afirman que las personas forman sus componentes cognitivos de actitud, por lo menos parcialmente, con base en el aprendizaje sobre los atributos de los objetos. Y, así, colocan en los objetos atributos adicionales que son valorados coherentemente con sus actitudes y expectativas existentes. Una vez que la evaluación del objeto y las actitudes son derivadas positivamente y que también son coherentes con las creencias existentes sobre el objeto.

Esta evolución cognitiva confirma la coherencia de las creencias y aumenta el nivel de la confianza (Smith; Swinyard, 1988). En este caso, el efecto de la evaluación positiva de este particular comportamiento va a reforzar las creencias positivas en relación al objeto de actitud y así, la satisfacción y la lealdad a la marca aumentan, lo que determina probabilidad de regreso y de tolerancia a determinado precio. Recientemente, Back (2005) confirmó, con base en su estudio impírico en el sector de hotelero, que parte de la satisfacción del cliente es consecuencia del estado de fidelidad del cliente al produto o servicio.

A seguir las hipótesis llevadas en consideración en esta investigación:

H1: la personalidad de la marca está associada positivamente con las emociones positivas del cliente;

H2: la personalidad de la marca está negativamente asociada con las emociones negativas del cliente;

H3: la emoción positiva asociada con a satisfacción del cliente positivamente;

H4: emoción negativa está asociada con la satisfacción del cliente negativamente;

H5: la emoción positiva está asociada con la lealtad a la marca positivamente;

H6: emoción negativa está asociada con la lealtad negativamente;

H7: la satisfacción del cliente está positivamente asociada con la fidelidad a la marca. 


\section{Las variables}

Se desarrolló un cuestionario baseado en revisión bibliográfica y en un estudio central. Para garantizar la confiabilidad y la validad de las escalas, también fueron realizados testes. Relacionados a la personalidade de los restaurantes fueron agrupados 42 ítenes baseado en el estudio de Aaker (1997) referentes a la personalidad y a la marca. Son identificados en términos de cinco dimensiones: la sinceridad, la excitación, la competencia, la sofisticación y robustez.

Se conferió la escala de emoción en cinco ítenes positivos - contento, satisfecho, animado, feliz y muy satisfecho - y en cinco puntos negativos - irritado, descontento, ignorado, irritado y ansioso -, habiéndose considerado 1 para "no estoy de acuerdo" a 5 para "concuerdo".Yoo et al. (1998) realizó entrevistas etnográficas para identificar la emoción de los clientes en relación a las respuestas que caracterizan la experiencia individual. El estudio identificó cinco puntos positivos - placer, atrayente, animado, contento y feliz - y cinco puntos negativos - ignorado, ansioso, anulado, descontento e irritado. En este estudio, en razón del direccionamento para el sector de restaurantes típicos de turismo, se acrescentó un iten más para capturar el grado de satisfacción de los clientes al experimentar una sensación de saciedad después de la comida.

En relación a la satisfacción del cliente se adoptó tres escalas de satisfacción de acuerdo con Lee, Lee; Yoo (2000). Siguiendo la linea con Westbrook y Oliver (1991), la satisfacción del cliente es mayor en la cena de que en relación al almuerzo en términos de sus expectativas. La evaluación de acuerdo con el tema “ yo me siento satisfecho con mi decisión de cenar en el restaurante", con las posibilidades de respuestas (1. no estoy de acuerdo a 5. estoy de acuerdo). El segundo item, de preguntas: "Cual es la satisfacción percibida con la cena en el restaurante". La escala de evaluación de como él se siente en relación al restaurante (1. muy malo a 5 . muy bueno).

La escala de lealtad fue elaborada por medición en cuatro ítenes con base en (Zeithaml et al. 1996). Los ítenes medidos de acuerdo con una escala de 5 puntos de tipo Likert (1. fuertemente no estoy de acuerdo a 5 . estoy plenamente de acuerdo), con declaraciones sobre la intención de decir cosas positivas para los otros, tales como: recomendar el restaurante para otro cliente; presentar una fuerte intención de regresar a este restaurante y también agregar ciertas observaciones, como por ejemplo: escojo este restaurante en primero lugar en relación a los otros restaurantes.

El estudio fue realizado con 540 clientes que habían cenado en los restaurantes típicos de Campos de JordãoSP - Brasil escojidos para el estudio. El tipo de restaurante es reconocido nacionalmente como establecimiento para familias y con precio medio para almuerzo y cena. La investigación fue realizada en seis restaurantes convenientemente seleccionados con base, no sólo en la localización y volumen de negocios, fue también en función de su facturamiento y de la equivalencia de los servicios durante el período de un mes.

Las entrevistas fueron presentadas a los clientes durante el almuerzo y la cena todos los días de la semana. Un total de 90 entrevistas fueron realizadas entre los clientes en cada uno de los restaurantes mientras estaban en el local - sumando un total de 540 cuestionarios -, siendo que 512 fueron devolvidos. El número de respuestas a ser utiizadas fue de 480, obteniendo un $89 \%$ de respuestas. Este alto porcentaje de respuesta se devió a un descuento ofrecido para los participantes de la investigación en la próxima visita al restaurante. Los datos del universo de esta investigación son mostrados en el Cuadro 1, a seguir:

\begin{tabular}{|c|l|c|c|}
\hline & CARACTERÍSTICAS & RESPUESTAS & PORCENTAJE \% \\
\hline & & & \\
\hline Sexo & Hombres & 214 & 44.6 \\
\hline & Mujeres & 266 & 55.4 \\
\hline & & & \\
\hline Edad & Menos de 24 & 112 & 23.4 \\
\hline & De 25 a 30 & 160 & 33.3 \\
\hline & Más de 30 & 208 & 43.3 \\
\hline & & & \\
\hline Escolaridad & Post-graduación & 130 & 27.1 \\
\hline & Superior & 295 & 61.5 \\
\hline & Secundaria & 55 & 11.4 \\
\hline
\end{tabular}

Cuadro 1. Muestra demográfica de los participantes. Fuente. Datos del estudio

Los coeficientes alfas de dimensiones positivas y negativas fueron 0.763 y 0.908 , respectivamente. Los coeficientes de sinceridad, entusiasmo, competencia, sofisticación y robustez fueron $0.817,0.810,0.883,0.834$ y 0.780 , respectivamente. El coeficiente alfa para el coeficiente satisfacción del cliente fue de 0.886 y el coeficiente alfa de lealdad fue 0.910 .

\section{Análisis y resultado de la investigación}

El resumen del perfil demográfico de los entrevistados presentados en el Cuadro 1 muestra que la mayoría que respondieron a las preguntas son del sexo femenino (55.4\%), con edad de más de 30 anos (43.3\%) y con curso superior (61.5\%). El análisis factorial y test de con- 
fiabilidad en un procedimiento de rotación varimax fue utilizada para identificar dimensiones subyacentes en relación a la personalidad de la marca. En seguida, el test de confiabilidad fue utilizado para testar la consistencia interna para las variables, emoción, satisfacción y lealtad.

Como muestra el Cuadro 2, el análisis factorial exploratorio con rotación varimax para la personalidad de la marca del restaurante reveló cinco factores con base en un corte de autovalor de 1 . Esos cinco factores explicaron un total acumulado de $74.02 \%$ de la variante en los datos. La interpretación de esos factores está en las siguientes dimensiones de la marca de personalidad de los restaurantes (las variaciones están mensionadas entre paréntesis): competencia (17.69\%), robustez (15.82\%), sinceridad (15.61\%), excitación (12.61\%), y sofisticación (12.9\%).

\section{El modelo de medición}

La medición de la cualidad total fue evaluada utilizando análisis factorial confirmatoria (Anderson; Gerbing, 1992). Mismo que la cualidad de medición sea evaluado factor por factor, cada indicador del múltiplo íten fue considerado simultáneamente para garantizar la máxima validad del test convergente y discriminante deberían ser retirados a partir del análisis adicional (ver Cuadro 3).

Todas las cargas que ultrapasen 0.5 , y cada indicador $\mathrm{t}$ con valor superior a $12.7(\mathrm{p}<0,001)$. El factor $\mathrm{X}^{2}$ de ajuste estadístico muestra 503.05 con 21 grados de liberdad $(p<0,001)$. La raíz cuadrada con erro de aproximación - (Root Mean Square Error of Approximation - RMSEA) es de 0.045, y el índice de ajuste comparativo (IAC) es de 0.96, y el índice ajustado de bondad es de

\begin{tabular}{|l|c|c|c|c|c|c|}
\hline \multicolumn{1}{|c|}{$\begin{array}{c}\text { FACTOR } \\
\text { DE } \\
\text { CARGA }\end{array}$} & $\begin{array}{c}\text { AUTO } \\
\text { VALOR }\end{array}$ & VARIACIÓN & $\begin{array}{c}\text { NOMBRE } \\
\text { DE } \\
\text { FACTOR }\end{array}$ & $\begin{array}{c}\text { CORRELACIÒN } \\
\text { TOTAL }\end{array}$ & $\alpha$ \\
\hline Negocio & 0.81 & 2.89 & 17.69 & Competencia & 0.70 & 0.81 \\
\hline Suceso & 0.79 & & & & 0.69 & \\
\hline Técnica & 0.81 & & & & 0.72 & \\
\hline Esfuerzo & 0.87 & & & & 0.75 & \\
\hline Confianza & 0.73 & & & & 0.64 & \\
\hline Robusto & 0.66 & 2.69 & 15.82 & Robustez & 0.58 & 0.79 \\
\hline Resistencia & 0.79 & & & & 0.69 & \\
\hline Sincero & 0.88 & 2.66 & 15.61 & Sinceridad & 0.71 & 0.77 \\
\hline Honesto & 0.85 & & & & 0.75 & \\
\hline Realista & 0.75 & & & & 0.66 & \\
\hline Saludable & 0.89 & & & & 0.81 & \\
\hline Independiente & 0.71 & 2.22 & 12.61 & Excitación & 0.62 & 0.73 \\
\hline Único & 0.73 & & & & 0.71 & \\
\hline Jovial & 0.74 & & & & 0.59 & \\
\hline Encantador & 0.86 & & & & 0.73 & \\
\hline Femenino & 0.89 & 2.01 & 12.29 & Sofisticación & 0.79 & 0.75 \\
\hline Variedad & 0,81 & & & & 0.72 & \\
\hline Suavidad & 0.82 & & & & 0.71 & \\
\hline VARIACIÓn TOTAL & & & & & & \\
\hline
\end{tabular}

Cuadro 2. Resultados del análisis factorial y del test de confiabilidad de los participantes. Fuente. Datos de la investigación.

Se realizó un refinamiento en la escala y, también a través del análisis de correlación total para mejorar la confiabilidad de los resultados obtenidos. Esto condujo a la retención de 19 ítenes, que representaban cinco dimensiones: factor de competencia (5 itens, $\alpha-0.81$ ), factor de robustez ( 4 itens, $\alpha-0.79$ ), factor de sinceridad (4 itens, $\alpha-0.77$ ), factor de excitación (3 itens, $\alpha-0.73$ ), y factor de sofisticación (3 itens, $\alpha-0.75$ ).
0.89, y el índice de ajuste de economía normalizada es de 0.77 . Todo soporte de apoyo a las estadísticas y a la cualidad de las mediciones globales fueron dadas debido al grande número de amuestra y del número de indicadores (Anderson \& Gerbing, 1992). Además, se verifica la evidencia de la validad discriminante cuando la proporción de variación extraida de cada participante excede el cuadrado del coeficiente $\Phi$ representando su correlación con otros factores.

Un par de escalas, con una alta correlación entre ellos es la satisfacción y la lealdad; con $\Phi=0.76 ; \Phi^{2}=0.57$ (ver en el Cuadro 4). Las varianciones extraidas estimadas son de 0,75 y 0,73 , respectivamente, indicando la validad discriminante adecuada. Mismo que se pueda también estar preocupado con la validad discriminante de los participantes de emociones positivas y satisfacción, la correlación entre las emociones positivas y satisfacción es de $0,65\left(\Phi^{2}=0,41\right)$. La estimativa extraida para esas escalas son 0.71 y 0.73 , respectivamente. Así, de acuerdo con esta evaluación, las medidas parecen tener niveles aceptables y con validad.

\section{Test de las hipótesis}

En general, los resultados del modelo fueron analizados usando LISREL 8.5. Los términos estructurales de error (flechas) para las variables de emoción (positiva 


\begin{tabular}{|l|c|c|c|}
\hline \multicolumn{1}{|c|}{$\begin{array}{c}\text { PARTICIPANTES } \\
\text { VARIABLES }\end{array}$} & $\begin{array}{c}\text { PADRONIZACIÓN } \\
\text { FACTOR CARGA }\end{array}$ & $\begin{array}{c}\text { PARTICIPANTE } \\
\text { DE } \\
\text { CONFIANZA }\end{array}$ & MEDIAS** \\
\hline SINCERIDAD & & 0.69 & 0.44 \\
\hline Honestidad & $0.66(15,34)$ & & \\
\hline Realista & $0.3(13,77)$ & & \\
\hline Saudável & $0.61(13,31)$ & & \\
\hline EXCITANTE & & 0.71 & 0.51 \\
\hline loven & $0.68(16,63)$ & & \\
\hline Único & $0.64(13,87)$ & & \\
\hline ndependiente & $0.77(17,34)$ & & \\
\hline COMPETENCIA & & 0.84 & 0.41 \\
\hline Trabajo & $073(16.94)$ & & \\
\hline Negocio & $0.70(16.55)$ & & \\
\hline Confianza & $0.69(16.40)$ & & \\
\hline Suceso & $0.75(17.02)$ & & 0.79 \\
\hline SOFISTICACIÓN & & 0.66 & 0.47 \\
\hline Atracción & $0.78(17.77)$ & & \\
\hline Femenidad & $0.61(14.94)$ & & \\
\hline EMOCIÓN + & & 0.87 & \\
\hline Contento & $0.81(21.67)$ & & 0.79 \\
\hline Placer & $0.73(20.91)$ & & \\
\hline EMOCIÓN - & $0.74(17.04)$ & & \\
\hline Descontento & & & \\
\hline Desplacer & $0.91(23.88)$ & & \\
\hline SATISFACCIÓN & & & \\
\hline Con el local & & & \\
\hline Sentimiento & & & \\
\hline FIDELIDAD & & & \\
\hline Regreso & & & \\
\hline Promover & & & \\
\hline
\end{tabular}

Cuadro 3.Modelo de medición resultante de la confirmación del Factor de Análisis. Fuente: Datos de la investigación. * . $\mathrm{X}^{2}=114.53(\mathrm{df}=57$, y valor de $\mathrm{p}=0,000)$, índice de ajuste $=0.94$, índice ajustado $=0.88$; erro de aproximação da raíz cuadrada $=0.068$; índice de ajuste normalizado $=0.97$ e índice de ajuste comparativo $=0.98$. ** variable media extraida.

\begin{tabular}{|c|c|c|c|c|c|c|c|}
\hline & 1 & 2 & 3 & 4 & 5 & MEDIA & SD \\
\hline $\begin{array}{c}\text { PERSONALIDAD DE LA } \\
\text { MARCA }\end{array}$ & 1.00 & & & & & & \\
\hline EMOCIÓN POSITIVA & $0.47^{*}$ & 1.00 & & & & 4.97 & 1.01 \\
\hline EMOCIÓN NEGATIVA & $(0.02)^{*}$ & $(0.16)^{*}$ & 1.00 & & & 2.79 & 1.41 \\
\hline SATISFACCIÓN DEL CLIENTE & $0.45^{*}$ & $0.3^{*}$ & $(0.30)^{*}$ & 1.00 & & 5.17 & 1.9 \\
\hline FIDELIDAD & $0.39^{*}$ & $0.59^{*}$ & $(0.18)^{*}$ & $0.74^{*}$ & 1.00 & 5.01 & 1.17 \\
\hline
\end{tabular}

Cuadro 4. Intercorrelaciones de los participantes, media y desvio padrón. Fuente: Datos de la investigación. y negativa) permitieron relacionar unos con los otros, pero no con cualquier otro termino de error estructural, porque estos dos representan las dos dimensiones emocionales del consumidor. La máxima verosimilitud de estimativas para los diferentes parámetros de ajuste global del modelo son presentados en el Cuadro 5.

$\mathrm{El}$ factor $\mathrm{X}^{2}$ sugiere que los datos de ajuste del modelo no fija para $\left(X^{2}=20.92\right.$; df $\left.83 ; p<0.01\right)$, porque la sensibilidad estadística de $\mathrm{X}^{2}$ no es una medida adecuada de ajuste del modelo. Entre tanto, la evaluación global del ajuste se basió en múltiplos indicadores (Bagozzi; Yi, 1988; Bollen, 1989; Hair et al., 1998). Pero, esos múltiplos indicadores sugirieron que el modelo tiene un buen ajuste, y que justifica la interpretación de los datos. El índice ajuste de bondad fue de 0.95, y el índice de ajuste normalizado fue de $0.96 \mathrm{y}$ el índice de ajuste no normatizado fue de 0.97. También, la correlación múltipla del cuadrado (CMC) fue de 0.059, y el índice de comparación (IC) fue de 0.97.

La correlación múltipla del cuadrado $\left(\mathrm{CMC} ; \mathrm{R}^{2}\right)$ para las ecuaciones estruturales indicados para satisfacción y lealdad fueron altas. Más de la mitad de la variación $(\mathrm{CMC}=0,69)$ en lealdad fue explicado por el efecto directo en las emociones positivas y negativas y de la satisfacción y sus efectos indirectos en las dimensiones de personalidad de la marca, tales como sinceridad, entusiasmo, competencia, sofisticación y robustez. Para la satisfación del cliente $(\mathrm{CMC}=0,60)$, y la variación fue explicada por los efectos directos de emociones positivas y negativas y las dimensiones de la personalidad de la marca.

Para la variación positiva de emoción $(\mathrm{CMC}=, 32)$, la variación fue explicada por los

efectos directos de las dimensiones a la personalidad de la marca. Por lo tanto, la variación negativa de emoción $(\mathrm{CMC}=0.02)$ por la personalidad de la marca no fue suficiente. Los Cuadros 5 y 6 presentan los resultados y estimativas de los parámetros normatizados.

La personalidad de la marca H1 (hipótese 1) tiene un efecto positivo sobre la emoción positiva $(\mathrm{Y} 11=0,57$; valor $\mathrm{t}=8,76)$, estadísticamente con significativo nivel $\mathrm{p}$ $<0,01 \mathrm{y}$, por lo tanto, confirma la H1. Este resultado fue consistente con la conclusión de Izard et al. (1993) que la personalidad de la marca es positivamente relacionada con la emoción positiva. Como todos los atributos de la personalidad de la marca tienen connotaciones positivas, hubo un impacto positivo de la personalidad de la marca con la emoción positiva. Los clientes perciben la marca de un 


\begin{tabular}{|l|c|c|}
\hline \multicolumn{1}{|c|}{ ELEMENTOS } & $\begin{array}{l}\text { MODELO } \\
\text { PROPUESTO }\end{array}$ & $\begin{array}{c}\text { MODELO } \\
\text { COMPETIDORES }\end{array}$ \\
\hline PERSONALIDAD DE LA MARCA & & \\
\hline Emoción positiva & $0.56(8.76)$ & $0.60(9.44)$ \\
\hline Emoción negativa & $-0.13(-2.29)$ & $-0.13(-2.35)$ \\
\hline Satisfacción & $0.27(4.56)$ & $0.22(4.41)$ \\
\hline Lealdad & $0.09(0.11)$ & $0.05(0.09)$ \\
\hline EMOCIONES POSITIVAS & & \\
\hline Satisfacción & $0.7(11.13)$ & $0.68(14.33)$ \\
\hline Lealdad & $0.15(2.07)$ & $0.14(2.09)$ \\
\hline EMOCIONES NEGATIVAS & & \\
\hline Satisfacción & $-0.15(-3,38)$ & $-0.13(-2,93)$ \\
\hline Lealdad & $-0.10(-3,29)$ & $-0.11(-2,81)$ \\
\hline EFECTOS INDIRECTOS & & \\
\hline Satisfacción a la marca & $0.37(6,93)$ & $0.47(8.59)$ \\
\hline Fidelidad a la marca & $0.53(9,41)$ & $0.41(8.43)$ \\
\hline Fidelidad a las emociones & $0.43(7,16)$ & $0.55(8.51)$ \\
\hline EFECTOS TOTALES & & \\
\hline Emociones positivas a la marca & $0.59(8,88)$ & $0.61(9.47)$ \\
\hline Emociones negativas a la marca & $-0.15(-2.31)$ & $-0.13(-2.33)$ \\
\hline Satisfacción a la marca & $0.61(9.55)$ & $0.47(8.71)$ \\
\hline Fidelidad a la marca & $0.59(9,37)$ & $0.42(8.39)$ \\
\hline Lealdad & $0.77(11.67)$ & $0.75(11.98)$ \\
\hline Emociones de fidelidad & $0.61(9.97)$ & $0.69(10.33)$ \\
\hline Satisfacción general & $0.79(11.53)$ & $0.82(13.13)$ \\
\hline
\end{tabular}

Cuadro 5. Parámetros normatizados. Fuente: Datos de la investigación.

\begin{tabular}{|c|c|c|}
\hline CMR (R) & $\begin{array}{c}\text { MODELO } \\
\text { PROPUESTO }\end{array}$ & $\begin{array}{c}\text { MODELO } \\
\text { CONCURRENTE }\end{array}$ \\
\hline Emociones positivas & 0.34 & 0.39 \\
\hline Emociones negativas & 0.02 & 0.03 \\
\hline Satisfacción & 0.63 & 0.61 \\
\hline Fidelidad & 0.68 & 0.69 \\
\hline$\chi^{2}$ & 251.53 & 222.09 \\
\hline $\mathrm{df}$ & 81 & 83 \\
\hline $\mathrm{p}$ & 0.00 & 0.00 \\
\hline
\end{tabular}

Cuadro 6. Datos de la correlación múltipla del cuadrado. Fuente: Datos de la investigación.

Obs.:

a. $\chi^{2}=201.53 ; d f=81 ; p=.000 ; \mathrm{GFI}=.94 ; \mathrm{AGFI}=.92 ; \mathrm{CFI}=.96 ; \mathrm{NNFI}=.95 ;$ RMSEA $=.057$, b. $\chi^{2}=222.59 ; \mathrm{df}=82 ; p=.000 ; \mathrm{GFI}=.94 ; \mathrm{AGFI}=.91 ; \mathrm{CFI}=.96 ; \mathrm{NNFI}=.95 ; \mathrm{RMSEA}=.061$, c. $\chi^{2}=201.44 ; d f=80 ; p=.000 ; \mathrm{GFI}=.94 ; \mathrm{AGFI}=.92 ; \mathrm{CFI}=.96 ; \mathrm{NNFI}=.95 ; \mathrm{RMSEA}=.058$, $t$ crit $\sigma=0.05=1.96$. $t$ crit $\sigma=0.01=2.58$.

restaurante honesto, real, y saludable, que genera una emoción positiva. Así, la $\mathrm{H} 2$ fue confirmada, mostrando un efecto negativo y significativo de la personalidad de la marca en emociones negativas $(\mathrm{Y} 11=-0,13$; valor $\mathrm{t}$ $=-2,29)$ que, también, fue consistente con resultados de estudios anteriores de Costa y McCrae (1991). Las H3 y
H4 fue confirmada, pues las emociones positivas y negativas fueron asociadas con la satisfacción del cliente. La emoción positiva del cliente sobre el restaurante tuvo un efecto positivo y muy significativo en su satisfacción ( $331=0.57$; valor $\mathrm{t}=11.13 ; \mathrm{p}<0.01)$, apoyando así la H3. De otro modo, la emoción negativa tuvo un efecto negativo sobre la satisfacción del cliente $(B 32=-0.15$; valor $\mathrm{t}=-3.38 ; \mathrm{p}<0.01)$, apoyando así la $\mathrm{H} 4$. Las $\mathrm{H} 3$ y $\mathrm{H} 4$ fueron consistentes con las conclusiones de Oliver (1997) sobre el proceso de dupla satisfacción de los clientes.

Como Oliver (1997) afirma, la satisfacción resulta de un duplo proceso un cognitivo y otro afectivo. Cuando los clientes cognitivamente evaluan el desempeño de una marca de restaurante, ellos tienen una memoria positiva que, en seguida, lconduce a un efecto positivo. Como los clientes desarrollan cognitivamente la performance sobre la marca del restaurante, pasan a tener una memoria que conduce con afectividad los efectos positivos y la combinación de esos dos procesos genera una influencia afectiva directa sobre su satisfacción. Las H5 y H6 confirmó que tanto las emociones positivas y negativas fueron asociadas con la fidelidad a la marca. La emoción positiva para algunos restaurantes de la investigación tuvo un efecto positivo muy significativo sobre la fidelidad a la marca $(\beta 41=0,15$, valor $\mathrm{t}=2.31$; $\mathrm{p}<0.05)$; así, la H5 fue comprobada. Contrariamente a las expectativas, una emoción negativa no tiene un efecto significativo sobre la lealtad ( $\$ 42=-0.10$; valor $\mathrm{t}=$ -3.29). Ese resultado indicó que las emociones negativas no desempeñan un papel suficiente para reducir el nivel de fidelidad a la marca.

Las emociones negativas reprimidas en algunos casos tienen variaciones para emoción positiva, que fue irrelevante para la fidelidad a la marca. Esto es posible debido a los problemas de multi linearidad y el estado de correlación entre emociones positivas y negativas $(r$ $=-0.16 ; \mathrm{p}<0.01$ ), acompañada por la significativa correlación entre emociones positivas y lealdad $(r=0.59 ; p$ $<0.01)$ y la correlación negativa significativa entre emociones negativas y lealtad $(r=-0.18$; $\mathrm{p}<0.01)$, condujo la supresión de efectos conforme (Maassen; Bakker, 2001). La $\mathrm{H} 7$ fue confirmada, visto que la satisfacción fue positivamente asociada con la lealtad a la marca del restaurante. Como esperado, la satisfacción con la marca del restaurante tuvo un efecto positivo significativo sobre la lealtad ( $(43=0.7$; valor $\mathrm{t}=11.7$; $\mathrm{p}<0.1$ ). En base en estos resultados, la satisfacción del cliente influenció positivamente la lealtad a la marca, al paso que las informaciones contenidas por los clientes sobre la marca fueron superiores al que es conocido sobre la oferta de la competencia. Como el cliente puede tener 
sentimentos positivos de satisfacción y placer en relación lo que la marca ofrece y desempeña y eso contribuye para tornalos fieles a la marca, manteniendo un compromiso constante con la marca específica.

Análisis adicionales de los efectos indirectos fueron conducidas para investigar si la personalidad de la marca tuvo un efecto sobre la satisfacción y lealdad a través del papel de mediación en las emociones del cliente. La personalidad de la marca tuvo un efecto positivo indirecto sobre la satisfacción $(0.37$, valor $\mathrm{t}=6.93 ; \mathrm{p}<0.01)$ $\mathrm{y}$ lealdad (0.53; valor $\mathrm{t}=9.41, \mathrm{p}<0.01)$. El efecto mediador de la emoción fue evidente en las ocasiones en que los clientes percibieron positivamente la personalidad de la marca, mostrando sus sentimentos positivos en relación a la marca y así, la satisfacción del cliente con la marca (restaurante). Además, los resultados empíricos demostraron que las emociones positivas tuvieron, también, un efecto positivo indirecto sobre la lealdad (0.37; valor $\mathrm{t}=6.93, \mathrm{p}<0.01)$.

Este factor es consistente con la conclusión del estudio de Ruyter y Bloemer (1999) que cuando un cliente tiene experiencia de afecto positivo en un restaurante, el cliente está dispuesto a regresar y repetir esa experiencia del servicio prestado y, por lo tanto, convertirse fiel al restaurante. Además, cuando un cliente tiene una experiencia negativa sobre un tipo específico de servicio, él deja de ser cliente de ese servicio y cambia para otra marca (Yu; Dean, 2001). Y, finalmente, una emoción negativa tuvo efecto negativo indirecto sobre la fidelidad (0.10 ; valor $\mathrm{t}=-3.29 ; \mathrm{p}<0.01$ ), mismo que venga a tener un efecto directo y positivo en la lealdad por variables supresoras.

\section{Implicaciones del estúdio}

Las emociones de los clientes desempeñan un papel dominante en la explicación de la satisfacción, confianza, y fidelidad del cliente. Mismo que algunas investigaciones hayan sugerido que la personalidad de la marca Lee et al. (BIEL, 1993), aumenta el nivel de confianza y lealdad y también aumenta la preferencia de los clientes en utilizar determinados tipos de servicios ofrecidos por el restaurante. (Sirgy, 1982). Entre tanto, este estudio sugiere que las emociones de los clientes parecen ser percibidas en las preguntas esenciales dentro del contexto de servicios, especialmente, en el sector de restaurantes típicos.

Esta investigación provee evidencia empírica para el desarrollo del comportamiento que envuelven los ítenes en relación a la fidelidad de la marca del restaurante, las emociones positivas y negativas y a la satisfacción del cliente con la marca. Los efectos de la emoción y de la satisfacción del cliente en la relación entre la personalidad de la marca y la lealdad a la marca fueron significativas.

Los resultados confirman los efectos positivos de la personalidad de la marca en la emoción positiva y en la satisfacción del cliente, bien como con su fidelidad (Barbin; Darden, 1996; Ruyter; Bloemer, 1999; Yik, 2001). Los resultados apoyaron la conclusión que los clientes perciben en la personalidad de la marca positivamente y aumenta su grado de "placer" en relación a ella. A su vez, los resultados indican, también, una intención positiva en el compromiso de regresar sempre al restaurante. Por lo tanto, este estudio afirma la importancia de medir la personalidad de la marca en la percepción de clientes y su fidelidad al restaurante y otorga, también, una mejor comprensión de su comportamiento ante el negocio restaurante.

Además, confirma las cinco dimensiones de la personalidad de la marca de un restaurante: sinceridad, excitación, competencia, sofisticación y robustez, como en el estudio de Aaker (1997). Por ejemplo, el factor sinceridad consiste de cuatro elementos: ambiente sincero, honesto, saludable y alegre, de paso que el factor sinceridad en este estudio está constituido también por cuatro diferentes elementos: sincero, honesto, real y saludable. También, el factor emoción consiste también de cuatro aspectos: audaz, espirituoso, imaginativo y actualizado, de paso que el factor emoción consiste de tres vertientes: independiente, original e innovativo.

Los resultados de esta investigación también fueron consistentes con Yik (2001) en la demonstración que la personalidad de la marca tiene un impacto significativo sobre la emoción individual de las personas e influencia en su comportamiento de consumo. Especifícamente, cuando los clientes establecieron que la personalidad de la marca de un restaurante como un servicio diferenciado, pasan a tener un determinado nivel de expectativa sobre el mismo en relación a la marca. La expectativa de la marca lleva a una respuesta emocional en la mente del cliente, resultando en un generador de emociones positivas o negativas. Estos resultados son consistentes con estudios anteriores en que la emoción negativa también fue un fuerte orientador de la fidelidad a la marca.

Así, se vuelve lógico para los emprendedores de restaurante en analizar los clientes que tienen experiencias emocionales negativas separadamente de los clientes que poseen experiencias emocionales positivas. Así, los emprendedores pueden tomar medidas inmediatas para controlar los problemas relacionados com situaciones que puedan crear experiencias emocionales negativas a los clientes.

Una mejor comprensión del desarrollo de las emo- 
ciones puede permitir a los emprendedores aumentar y mejorar el relacionamiento con los clientes y ampliar la ocupación de sus mesas. Los resultados del presente estudio sugieren también que los emprendedores de restaurantes típicos y turísticos deban desarrollar marketing y sistemas de información que monitoren continuamente las percepciones de los clientes y la imagen de la marca del restaurante. Específicamente, los emprendedores deben examinar como es percibida la personalidad de la marca ante sus clientes y verificar si esa percepción está em línea con la misión, visión y objetivo del restaurante.

La Teoría de Auto Imagen indica que los consumidores prefieren los productos, marcas, tiendas, restaurantes y otros servicios comerciales, cuya percepción psicológica de sus características sea congruente con sus percepciones y características psicológicas. Además, el marketing del objetivo selectivo debe ser cuidadosamente considerado a la luz de la personalidad de la marca del restaurante y de su servicio. Como los servicios de restaurante son caracterizados por intangibilidad, la personalidad de la marca consistente con la imagen percibida por el conjunto de su servicio tangibilidad por lo productos físicos ofrecidos, la atención de los emprendedores debe ser redobrada en un mercado altamente competitivo. Esto es especialmente verdadero para el segmento de mercado de lujo, en que los clientes compran no sólo los productos, mas también, los símbolos de lujo, orgullo y status.

\section{Limitaciones y futuro de investigación}

La presente investigación tiene diversas limitaciones. En primer lugar, los resultados no pueden ser generalizados para otros segmentos de restaurante. Estos datos fueron colectados de clientes en restaurantes típicos de región turística y familiar. Así como también, la selección de la muestra para este estudio no fue puramente aleatória. Como se observa, la muestra aleatoria es poco desarrollada en este sector, por lo tanto, incluir una mayor variedad de tipos diferentes de restaurante y de segmentos aumentaria la validad de la investigación.

Por lo tanto, futuros estudios deberan ser desarrollados en un proyecto sistemático que mejor represente ese universo. Por consiguiente, este estudio buscó presentar una amplia base, teóricamente guiada en trabajo de campo de los efectos de la personalidad de la marca, los estados emocionales de los clientes, las influencias en su satisfacción y como se vuelven fieles a la marca (restaurante). Los resultados son reveladores e indican la posibilidad de futuras investigaciones que permitirán ampliar y mejorar la estructura y servicios de los res- taurantes. Futuras investigaciones deberán ser incluidas para avaliar: medidas comportamentales de lealdad a la marca, los efectos de la personalidad del restaurante en el estado emocional de los clientes, lo que conduce a los clientes a la satisfacción, lealdad y fidelidad.

Sería útil también, examinar los efectos de la personalidad de auto congruencia em el estado emocional de los clientes, su satisfacción y lealdad con el restaurante. Finalmente, una mayor variedad de tipos y localizaciones de restaurantes debe ser estudiada para ampliar y fortalecer estos resultados. Diferentes categorías y tipos de restaurantes pueden tener diferentes personalidades de marcas y pueden tener diferentes relaciones con satisfacción y fidelización de los clientes a la marca.

\section{Referencias}

Aaker, D. A.

1996. "Measuring brand equity across products and markets". California Management Review, 38(3): 102-120.

Aaker, J. L.

1997. "Dimensions of brand personality". Journal of Marketing Research,34(3): 347-356.

Anderson, J. C.; Gerbing, D. W.

1992. "Assumptions and comparative strengths of the two-step approach". Sociological Methods and Research, 30(3): 321-333.

Back, K. J.

2005. "The Effects of Image Congruence on Hotel Customers' Brand Loyalty". Journal of Hospitality and Tourism Research, 28(4): 448-467.

Bagozzi, R. P., Gopinath, M.; Nyer, P. U.

1999. "The role of emotions in marketing". Journal of the Academy of Marketing Science, 27(2): 184-206.

Bagozzi, R. P.; YI, Y.

1988. "On the evaluation of structural equation models". Journal of the Academy of Marketing Science, 16(1): 74-94.

Baker, J., Grewal, D.; Levy, M.

1992. "An experimental approach to making retail store environmental decisions". Journal of Retailing, 68(4): 45-460.

Barsky, J. D.; Nash, L.

2002. "Evoking emotion: Affective keys to hotel loyalty". Cornell Hotel and Restaurant Administration Quarterly, 43(1): 39-46.

Batra, R.; Holbrook, M. B.

1990."Developing a typology of affective responses to advertising". Psychology and Marketing 7: 11-25.

Becker, C., Murrmann, S. K., Murrmann, K. F., \& Cheung, G. W.

1999. "A pan-cultural study of restaurant service expectations in the United States and Hong Kong". Journal of Hospitality \& Tourism Research, 24(4): 526538. 
Biel, A.

1993. "Converting image into equity". In D. A. Aaker \& A. L. Biel (Eds.), Brand equity and advertising (pp. 67-81). Hillsdale, NJ: Lawrence Erlbaum.

Bitner, M. J.

1990. "Evaluating service encounters: The effects of physical surroundings and employee responses". Journal of Marketing, 54(2): 69-82.

Bollen, K. A.

1989. Structural equations with latent variables. Toronto, Ontario,Canada: Wiley.

Burke, B.

1994. Position, personality, not price, should frame consumer messages. Brandweek, p.35-36. Burt, J.

2010. "Opinion: Jaguar needs a new meow". The San Diego Union-Tribune. http://www3.signonsandiego. com/news/business/ 05/13/2010.

Cronin, J. J.; Taylor, S. A.

1992. "Measuring service quality: A reexamination and extension". Journal of Marketing, 56(3): 55-68.

D’astous, A.; Lévesque, M.

2003. "A scale for measuring store personality". Psychology \& Marketing, 20(5): 455-469.

Davidow, W. H., \& Uttal, B.

1989. Total customer service: The ultimate weapon. New York: Harper \& Row.

Ruyter, K., \& Bloemer, J.

1999. "Customer loyalty in extended service settings: The interaction between satisfaction, value attainment and positive mood". International Journal of Service Industry Management, 10(3): 320-336.

Desai, K. K.; Mahajan, V.

1998."Strategic role of affect-based attitudes in the acquisition, development, and retention of customers". Journal of Business Research, 42(3): 309-324.

Dubé, L.; Menon, K.

1993."Multiple roles of consumption emotions in postpurchase satisfaction with extended service transactions". International Journal of Service Industry Management, 11(3): 287-304.

Eagly, A. C.; Chaiken, S.

1993. The psychology of attitudes. Dallas, TX: Harcourt.

Faria, M.S.A; Tachizawa, T.

2008. Criação de novos negócios:gestão de micros e pequenas empresas. São Paulo. FGV.

Fornell, C., Johnson, M. D., Anderson, E. W., Cha, J.;\& Bryant, B. E.

1996. "The American customer satisfaction index: Nature, purpose, and findings". Journal of Marketing, 60(4): 7-18.

Gilbert, G. R.; Veloutsou, C.

2006."A cross-industry comparison of customer satisfaction”. Journal of Services Marketing, 20(5): 298-308.

Hair, J. F., Anderson, R. E., Tatham, R. L.; Black, W. C. 1998. Multivariate data analysis. Upper Saddle River, NJ: Prentice Hall.
Han, H. \& Back, K. J.

2007."Investigating the effects of consumption emotions on customer satisfaction and repeat visit intentions in the lodging industry". Journal of Hospitality Marketing \& Management, 15(3): 5-30.

$\mathrm{Hsu}, \mathrm{C}$.

2009. Hospitality and tourism education in Asia-Pacific: Trends,developments and a look into the future. Paper presented at 2009 I-CHRIE Conference, San Francisco, CA.

Izard, C. E., Libero, D. Z., Putnam, P.; Haynes, O. M.

1993."Stability of emotion experiences and their relations to traits of personality". Journal of Personality and Social Psychology, 64(5): 847-860.

Jiang, W., Dev, C. S.; Rao, V. R.

2002."Brand extension and customer loyalty". Cornell Hotel and Restaurant Administration Quarterly, 43(1): 5-16.

Larson, D.

2002."Building a brand's personality from the customer up". Direct Marketing, 65(6): 17-21.

Laverie, D., Kleine, R. E., Iii; Kleine, S. S.

1993. Linking emotions and values in consumption experiences: An exploratory study. in Advances in Consumer Research, Vol. 20, ed. Leigh McAlister and Michael L. Rothschild, Provo, UT: Association for Consumer Research, p.70-75.

Lee, C. K., Lee, Y. K.; Lee, B. K.

2005."Korea's destination image formed by the 2002 World Cup". Annals of Tourism Research, 32(4): 839858.

Lee, H., Lee, Y.; Yoo, D.

2001."The determinants of perceived service quality and its relationship with satisfaction". Journal of Services Marketing, 14(3): 217-231.

Maassen, G. H.; Bakker, A. B.

2001."Suppressor variables in path models: Definition and interpretation”. Sociological Methods \& Research, 39(2): 241-270

Machleit, K.A.; Eroglu, S.A.

2000."Describing and measuring emotional response to shopping experience". Journal of Business Research, 49: 101-111.

Mattila, A. S.; Enz, C. A.

2002."The role of emotions in service encounters". Journal of Service Research, 4(4): 268.

Mattila, A. S., \& Patterson, P. G.

2004. "Service recovery and fairness perceptions in collectivist and individualist contexts".

Journal of Service Research, 6(4), 336-346.

McCrae, R. R., Costa, P.T., Ostendorf, F., Angleitner, A., Hrebícková, M., Avia, M.D., et al.

2000."Nature over nurture: temperament, personality, and life span development". Journal of Personality and Social Psychology, 78(1): 173-186.

Meyer, G. J.; Shack, J. R.

1989."Structural convergence of mood and personality: 
Evidence for old and new directions". Journal of Personality and Social Psychology, 57(4): 691-706.

Morris, J. D., Woo, C., Geason, J.; Kim, J.

2002."The Power of Affect: Predicting Intention”. Journal of Advertising Research, 42(3): 7-17.

$\mathrm{Oh}, \mathrm{H}$.

2005."Measuring affective reactions to print apparel advertisements: A scale development". Journal of Fashion Marketing and Management, 9(3), p.283-305.

Oliver, R. L.

1997. Satisfaction: A behavioral perspective on the consumption. New York: McGraw-Hill.

Parasuraman, A., Zeithaml, V. A.; Berry, L. L.

1994."Reassessment of expectations as a comparison standard in measuring service quality: Implications for further research". Journal of Marketing, 58(1): 111-124.

Park, B.

1986."A method for studying the development of impressions in real people". Journal of Personality and Social Psychology, 51(5): 907-917.

Plutchik, R.

1984."Emotions: A general psychoevolutionary theory". In K. R. Scherer \& P. Ekman Approaches to emotion (p.197-219). Hillsdale, NJ: Lawrence Erlbaum.

Randall, G.

1997. Branding, marketing in action series. London: Kogan Page.

Rust, R.; Zahorik, A. J.

1993."Customer satisfaction, customer retention, and market share". Journal of Retailing, 69(2): 193-215.

Siguaw, J. A., Mattila, A.; Austin, J. R.

1999."The brand-personality scale: An application for restaurants". Cornell Hotel and Restaurant Administration Quarterly, 40(3): 48-55.

Smith, R. E.; Swinyard, W. R.

1988."Cognitive response to advertising and trial: Belief strength, belief confidence and product curiosity". Journal of Advertising, 17(3): 3-14.

Spreng, R. A., Mackenzie, S. B.; Olshavsky, R. W.

1996."A reexamination of the determinants of consumer satisfaction". Journal of Marketing, 60(3): 15-32.

Spreng, R. A.; Mackoy, R. D.

1996."An empirical examination of a model of perceived service quality and satisfaction". Journal of Retailing, 72(2): 201-214.

Walsh, K.

2000. "A service conundrum". Cornell Hotel and Restaurant Administration Quarterly, 41(5): 40-50.

Watson, D.; Clark, L. A.

1997."Extraversion and its positive emotional core". In R. Hogan, J. Johnson, \& S. Briggs, Handbook of personality psychology (p.767-793). San Diego, Academic Press.

Wilson, A.

2002."Attitudes towards customer satisfaction measurement in the etail sector". International Journal of
Market Research, 44(2): 213-222.

Yi, Y.

1990."A critical review of consumer satisfaction". In V.

A. Review of marketing (p.68-123). Chicago: American Marketing Association.

Yik, M. S. M.

2001."Predicting the big two of affect from the big five of personality". Journal of Research in Personality, 35(3): 247-277.

Yoo, C., Park, J.; Macinnis, D. J.

1998."Effects of store characteristics and in-store emotional experiences on store attitude". Journal of $\mathrm{Bu}$ siness Research, 42(3): 253-263.

Yuksel, A., Kilinc, U. K., \& Yuksel, F.

2006. "Cross-national analysis of hotel customers' attitudes toward complaining and their complaining behaviors". Tourism Management, 27(1): 11-24.

Zeithaml, V. A., Berry, L.; Parasuraman, A.

1996."The behavioral consequences of service quality". Journal of Marketing, 60(2): 31-46.

\section{APÉNDICE: Cuestionario utilizado en la investi- gación (en portugués)}

Questionário de Pesquisa de Satisfação dos Clientes

Restaurante Turístico típico e Familiar

Informações gerais sobre a pesquisa:

1. Leia atentamente a questão, antes de respondê-la através de um grau que corresponderá à sua satisfação ou nível de concordância com o requisito que está sendo avaliado;

2. Não há necessidade de se identificar na pesquisa, ou seja, não precisa escrever seu nome na folha; também não faça comentários com outros na hora da pesquisa nem posteriormente, sobre o quê você respondeu, porque cada pessoa é única em suas percepções;

3. Nós agradecemos sua importante participação para o processo de melhoria dos nossos serviços e produtos;

4. Para cada questão há somente uma resposta;

5. A Escala de Avaliação que será usada por você é de 1 a 5, onde:

(1) Discordo

(2) Discordo parcialmente

(3) Neutro

(4) Concordo parcialmente

(5) Concordo

Dados pessoais:

Idade ( ); Sexo ( );

Escolaridade: Secundário ( ); Superior Incompleto ( ); Superior completo ( ); Pós Graduação ( )

\section{Questionário:}

Parte 1: Personalidade da marca

1. O restaurante se caracteriza como um empreendimento voltado ao turista 
Discordo( ); Discordo parcialmente( ); Neutro( ); Concordo parcialmente( ); Concordo( )

2. O restaurante trata o cliente diferenciadamente de um negócio estritamente comercial

Discordo( ); Discordo parcialmente( ); Neutro( ); Concordo parcialmente( ); Concordo( )

3. O restaurante se esforça para atender plenamente os turistas

Discordo( ); Discordo parcialmente( ); Neutro( ); Concordo parcialmente( ); Concordo( )

4. Os funcionários do restaurante oferecem sinceridade em seus serviços

Discordo( ); Discordo parcialmente( ); Neutro( ); Concordo parcialmente( ); Concordo( )

5. O restaurante se caracteriza pela honestidade em suas promoções

Discordo( ); Discordo parcialmente( ); Neutro( ); Concordo parcialmente( ); Concordo( )

6. O ambiente do restaurante é jovial na percepção do turista

Discordo( ); Discordo parcialmente( ); Neutro( ); Concordo parcialmente( ); Concordo( )

7. O restaurante encanta o cliente que utiliza

Discordo( ); Discordo parcialmente( ); Neutro( ); Concordo parcialmente( ); Concordo( )

8. O restaurante sendo voltado para o turismo deve ser único

Discordo( ); Discordo parcialmente( ); Neutro( ); Concordo parcialmente( ); Concordo( )

9. O restaurante se caracteriza pela variedade de pratos regionais

Discordo( ); Discordo parcialmente( ); Neutro( ); Concordo parcialmente( ); Concordo( )

10.0 restaurante proporciona nova emoção ao turista

Discordo( ); Discordo parcialmente( ); Neutro( ); Concordo parcialmente( ); Concordo( )

11.0 restaurante se caracteriza como uma atração para o local

Discordo( ); Discordo parcialmente( ); Neutro( ); Concordo parcialmente( ); Concordo( )

12. O restaurante proporciona prazer ao turista

Discordo( ); Discordo parcialmente( ); Neutro( ); Concordo parcialmente( ); Concordo( )

13. O restaurante proporciona emoção ao turista

Discordo( ); Discordo parcialmente( ); Neutro( ); Concordo parcialmente( ); Concordo( )

14. O restaurante deve ser sofisticado

Discordo( ); Discordo parcialmente( ); Neutro( ); Concordo parcialmente( ); Concordo( )

15.0 restaurante proporciona uma forte excitação ao paladar

Discordo( ); Discordo parcialmente( ); Neutro( ); Concordo parcialmente( ); Concordo(

Parte 1: Sentimentos sobre a marca

1. Você tem uma satisfação positiva vindo ao restaurante

Discordo( ); Discordo parcialmente( ); Neutro( );
Concordo parcialmente( ); Concordo( )

2. Você tem lealdade com o restaurante, voltando sempre

Discordo( ); Discordo parcialmente( ); Neutro( ); Concordo parcialmente( ); Concordo( )

3. A marca do lhe proporciona satisfação

Discordo( ); Discordo parcialmente( ); Neutro( ); Concordo parcialmente( ); Concordo( )

4. Você é fiel ao restaurante

Discordo( ); Discordo parcialmente( ); Neutro( ); Concordo parcialmente( ); Concordo( )

5. Você é leal com a marca falando positivamente

Discordo( ); Discordo parcialmente( ); Neutro( ); Concordo parcialmente( ); Concordo( )

6. Você tem lealdade com o restaurante, voltando sempre

Discordo( ); Discordo parcialmente( ); Neutro( ); Concordo parcialmente( ); Concordo( )

7. A caracterização do atendimento e gentil e delicado

Discordo( ); Discordo parcialmente( ); Neutro( ); Concordo parcialmente( ); Concordo( )

8. O restaurante lhe proporciona sentimento para voltar sempre

Discordo( ); Discordo parcialmente( ); Neutro( ); Concordo parcialmente( ); Concordo( )

9. Você tem lealdade com o restaurante, voltando sempre

Discordo( ); Discordo parcialmente( ); Neutro( ); Concordo parcialmente( ); Concordo( )

10.A uma forte emoção ao frequentar o restaurante

Discordo( ); Discordo parcialmente( ); Neutro( ); Concordo parcialmente( ); Concordo( )

11 . O restaurante lhe proporciona confiança em seu trabalho

Discordo( ); Discordo parcialmente( ); Neutro( ); Concordo parcialmente( ); Concordo( )

12.0 ambiente do restaurante é suave

Discordo( ); Discordo parcialmente( ); Neutro( ); Concordo parcialmente( ); Concordo( )

13. A localização é positiva

Discordo( ); Discordo parcialmente( ); Neutro( ); Concordo parcialmente( ); Concordo( )

14.0 restaurante em seu conjunto oferece plena satisfação

Discordo( ); Discordo parcialmente( ); Neutro( ); Concordo parcialmente( ); Concordo( )

15. Você recomendaria plenamente o restaurante para seus amigos e familiares

Discordo( ); Discordo parcialmente( ); Neutro( ); Concordo parcialmente( ); Concordo( )

Recibido:

$11 / 07 / 09$

Reenviado:

$20 / 12 / 10$

Aceptado:

$30 / 12 / 10$

Sometido a evaluación por pares anónimos 